UFTP 420/1996

\title{
Analyzing the nucleon spin in weak interaction processes at HERA energies
}

\author{
M. Maul, A. Schäfer \\ Institut für Theoretische Physik, J. W. Goethe Universität Frankfurt \\ 60054 Frankfurt am Main, Germany \\ e-mail: maul@th.physik.uni-frankfurt.de
}

\begin{abstract}
We investigate the possibility to measure the spin content carried by the different quark flavors in a nucleon by means of polarized deep inelastic scattering with $W^{ \pm}$ exchange at HERA. Such measurements require a polarized proton beam. The expected inclusive and semi inclusive asymmetries are sizable and for realistic luminosities the expected statistical accuracies are good enough to extract new and relevant information on the valence quark and the strange sea distributions.
\end{abstract}

PACS: 12.38.-t, 12.38.Qk, 13.15.Dk

Keywords: Quantum chromodynamics, experimental tests, charged-current reactions, polarized electrons and protons, HERA, semi inclusive measurements. 
Presently the realizability of a polarized proton beam at DESY allowing for polarized electron-proton scattering at $s \approx 10^{5}(\mathrm{GeV})^{2}$ is intensively discussed [1]. One of the novel possibilities opened by such experiments is the investigation of spin effects in charged current (CC) reactions, where the outgoing neutrino is detected by its missing momentum. CC events probe a different combination of spin-dependent quark-distribution functions than electromagnetic processes, thus allowing to extract information on the flavor decomposition of the polarized quark distribution functions. These experiments would yield results similar to those expected from semi inclusive fixed target experiments [2,3]. To put the full potential of such experiments into reality it is necessary to have polarized electron as well as positron beams at HERA. The most important property of $\mathrm{CC}$ reactions is that they distinguish quark and antiquark flavors, allowing to extract the polarized valence quark distributions $\Delta u_{V}$, $\Delta d_{V}$ as well as the strange ones $\Delta s, \Delta \bar{s}$. The latter two are usually assumed to be identical. If a difference between them were observed this could be related to the fact that the virtual coupling of $p$ to e.g. $\left(K^{+}+\right.$baryon $)$is stronger than to ( $K^{-}+$baryon). Including deep inelastic weak interaction processes, but neglecting lepton and quark masses the hadronic scattering tensor can be decomposed into eight structure functions [4]:

$$
\begin{aligned}
\frac{1}{2 m_{N}} W_{\mu \nu}= & -\frac{g_{\mu \nu}}{m_{N}} F_{1}\left(x, Q^{2}\right)+\frac{p_{\mu} p_{\nu}}{m_{N} p \cdot q} F_{2}\left(x, Q^{2}\right) \\
& +\frac{i \epsilon_{\mu \nu \alpha \beta}}{2 p \cdot q}\left[\frac{p^{\alpha} q^{\beta}}{m_{N}} F_{3}\left(x, Q^{2}\right)+2 q^{\alpha} S^{\beta} g_{1}\left(x, Q^{2}\right)-4 x p^{\alpha} S^{\beta} g_{2}\left(x, Q^{2}\right)\right] \\
& -\frac{p_{\mu} S_{\nu}+S_{\mu} p_{\nu}}{2 p \cdot q} g_{3}\left(x, Q^{2}\right)+\frac{S \cdot q}{(p \cdot q)^{2}} p_{\mu} p_{\nu} g_{4}\left(x, Q^{2}\right)+\frac{S \cdot q}{p \cdot q} g_{\mu \nu} g_{5}\left(x, Q^{2}\right)
\end{aligned}
$$

In the quark parton model $\left(g_{4}=0\right)$ the structure functions $g_{3}$ and $g_{5}$ fulfill in analogy to the Callan Gross relation $2 x g_{5}(x)=g_{3}(x)$ [5 8]. Contracted with the leptonic scattering tensor the differential cross section reads for the $\mathrm{CC}$ reactions with longitudinally polarized targets 


$$
\frac{d^{2} \sigma}{d x d Q^{2}}=\frac{G_{F}^{2} M_{W}^{4}}{4 \pi}\left(\left[a F_{1}-\lambda b F_{3}\right]+\left[-\lambda 2 b g_{1}+a g_{5}\right] \delta\right) \frac{1}{\left(Q^{2}+M_{W}^{2}\right)^{2}}
$$

where $G_{F}$ is Fermi's constant and $M_{W}$ the W-Boson mass. $\lambda$ is -1 for $e^{-}$and +1 for $e^{+}$, and $\delta$ denotes the longitudinal polarization of the nucleon antiparallel $(+1)$ or parallel $(-1)$ to the polarization of the lepton. The $y$ dependence is absorbed in the two constants $a$ and $b$

$$
a:=2\left(y^{2}-2 y+2\right) ; \quad b:=y(2-y) \quad .
$$

The asymmetry is defined by

$$
A:=\frac{d \sigma_{\uparrow \downarrow}-d \sigma_{\uparrow \uparrow}}{d \sigma_{\uparrow \downarrow}+d \sigma_{\uparrow \uparrow}}
$$

which leads to 10

$$
A^{W^{-}}=\frac{2 b g_{1}+a g_{5}}{a F_{1}+b F_{3}} ; \quad A^{W^{+}}=\frac{-2 b g_{1}+a g_{5}}{a F_{1}-b F_{3}} .
$$

The ratio of the factors $a$ and $b$ determines the contribution of the different structure functions to the asymmetry. For small $y$ values, for which the experimental statistics is best, $a$ is much larger than $b$.

In the framework of the quark parton model the eight combinations of distribution functions tested by CC asymmetries read [6]:

$$
\begin{array}{cl}
F_{1}^{W-}=u+c+\bar{d}+\bar{s}, & F_{1}^{W+}=d+s+\bar{u}+\bar{c}, \\
F_{3}^{W-}=2(u+c-\bar{d}-\bar{s}), & F_{3}^{W+}=2(d+s-\bar{u}-\bar{c}), \\
g_{1}^{W-}=\Delta u+\Delta c+\Delta \bar{d}+\Delta \bar{s}, & g_{1}^{W+}=\Delta d+\Delta s+\Delta \bar{u}+\Delta \bar{c} \\
g_{5}^{W-}=\Delta u+\Delta c-\Delta \bar{d}-\Delta \bar{s}, & g_{5}^{W+}=\Delta d+\Delta s-\Delta \bar{u}-\Delta \bar{c}
\end{array} .
$$

Rewriting (5) in terms of the parton distributions we get for the inclusive asymmetries: 


$$
\begin{aligned}
& A^{W^{-}}=\frac{\Delta u+\Delta c-\left[(y-1)^{2}\right][\Delta \bar{s}+\Delta \bar{d}]}{u+c+\left[(y-1)^{2}\right][\bar{s}+\bar{d}]}, \\
& A^{W^{+}}=\frac{\left[(y-1)^{2}\right][\Delta s+\Delta d]-\Delta \bar{u}-\Delta \bar{c}}{\left[(y-1)^{2}\right][s+d]+\bar{u}+\bar{c}} .
\end{aligned}
$$

In fig. 1 we show the expected asymmetries for HERA energies $\left(E_{l}=\right.$ $27.6 \mathrm{GeV}, E_{n}=820 \mathrm{GeV}$ ) with integrated cross sections in the $Q^{2}$ range $600-1000(\mathrm{GeV})^{2}$ and $1000(\mathrm{GeV})^{2}-$ maximum. We split the data into these two $Q^{2}$ ranges because it might not be obvious that the detection of missing momentum in the range $\sqrt{600} \mathrm{GeV}-\sqrt{1000} \mathrm{GeV}$ is absolutely reliable. (Usually already $Q^{2} \geq 600(\mathrm{GeV})^{2}$ is regarded as save). Fig. 1 shows that the low- $Q^{2}$ data do not dominate the statistics. So even if one would be very restrictive on the criteria for identifying CC events our conclusions would be hardly affected. Indeed, the situation has recently been improved. At present in $\mathrm{H} 1$ it is possible to control CC events with $p_{t}>15 \mathrm{GeV}$, i.e. $Q^{2}>225(\mathrm{GeV})^{2}$, so one could easily add another bin $[400-600](\mathrm{GeV})^{2}$ [9]. For the parton distributions we use the set "standard scenario, LO" given in [11]. The error bars are calculated according to

$$
\Delta A=\sqrt{\frac{1-A^{2}}{2 \mathcal{L} \Delta x d \sigma / d x}}
$$

where $\mathcal{L}$ denotes the luminosity, and $d \sigma$ is the unpolarized cross section. We assumed here the luminosity to be $100 \mathrm{pb}^{-1}$ and $100 \%$ polarization. This is identical to $200 \mathrm{pb}^{-1}$ and the beam polarization product $P_{e} P_{p}=0.5$. (Such a luminosity is planned if proton polarization is realized at HERA. For the far future, options to raise it to $1000 \mathrm{pb}^{-1}$ are discussed.) The remarkable features of our results are that the asymmetries are sizable and of considerable extent in the range $x \in[0.01,1]$, and that even for $100 \mathrm{pb}^{-1}$ the expected statistical accuracy for both $W^{+}$and $W^{-}$ exchange is rather good. 
In fig. 2 we compare the contribution of the sea quarks and valence quarks to the asymmetries using two different parton distribution sets. One is again the LO standard scenario [11], the other one is LO Gluon C [12]. The main distinction between the two sets is the fact that the polarized valence quark distribution given in [11] is nearly proportional to the unpolarized valence quark contribution, whereas in [12 the fit shows larger deviations. The total asymmetry is in [12] even larger than in [11]. Obviously the expected accuracy should allow to distinguish the two scenarios. Besides these single $W$ boson asymmetries one can form four combinations which are determined by pure valence contributions and by valence + sea contributions:

$$
A_{( \pm)_{1}( \pm)_{2}}:=\frac{\left(d \sigma_{\uparrow \downarrow}^{W^{-}}-d \sigma_{\uparrow \uparrow}^{W^{-}}\right)( \pm)_{1}\left(d \sigma_{\uparrow \downarrow}^{W^{+}}-d \sigma_{\uparrow \uparrow}^{W^{+}}\right)}{\left(d \sigma_{\uparrow \downarrow}^{W^{-}}+d \sigma_{\uparrow \uparrow}^{W^{-}}\right)( \pm)_{2}\left(d \sigma_{\uparrow \downarrow}^{W^{+}}+d \sigma_{\uparrow \uparrow}^{W^{+}}\right)} .
$$

Here the first \pm accounts for the numerator and the second for the denominator. With this definition we write:

$$
\begin{aligned}
& A_{++}=\frac{\Delta u_{V}+\left[(y-1)^{2}\right] \Delta d_{V}}{u_{T}+c_{T}+\left[(y-1)^{2}\right]\left[s_{T}+d_{T}\right]}, \\
& A_{--}=\frac{\Delta u_{T}+\Delta c_{T}-\left[(y-1)^{2}\right]\left[\Delta s_{T}+\Delta d_{T}\right]}{u_{V}-\left[(y-1)^{2}\right] d_{V}}, \\
& A_{-+}=\frac{\Delta u_{T}+\Delta c_{T}-\left[(y-1)^{2}\right]\left[\Delta s_{T}+\Delta d_{T}\right]}{u_{T}+c_{T}+\left[(y-1)^{2}\right]\left[s_{T}+d_{T}\right]}, \\
& A_{+-}=\frac{\Delta u_{V}+\left[(y-1)^{2}\right] \Delta d_{V}}{u_{V}-\left[(y-1)^{2}\right] d_{V}} .
\end{aligned}
$$

Here we defined for the quark distribution functions $q_{f}$

$$
\begin{aligned}
& \Delta q_{f}=q_{f, \uparrow \downarrow}-q_{f, \uparrow \uparrow} \quad, \\
& q_{f, V}=q_{f}-\bar{q}_{f} \quad, \\
& q_{f, T}=q_{f}+\bar{q}_{f} .
\end{aligned}
$$

While the inclusive asymmetries are mainly sensitive to the $\mathrm{u}$ - and $\mathrm{d}$ - quark flavor it is possible to extract information on the strange and antistrange sea by semi inclusive 
CC current measurements. As usual, we define the fragmentation function $D_{f}^{H}(z)$ which describes the probability that a quark with flavor $f$ fragments into a hadron $H$ which carries the energy fraction $z$ of the whole energy transferred to the nucleon. We want to discuss semi inclusive $\pi$ and $D$ production. As suggested in [13] we make the assumptions.

$$
\begin{aligned}
D_{1}^{\pi}(z) & =D_{u}^{\pi^{+}}(z)=D_{d}^{\pi^{-}}(z)=D_{\bar{d}}^{\pi^{+}}(z)=D_{\bar{u}}^{\pi^{-}}(z) \\
D_{2}^{\pi}(z) & =D_{d}^{\pi^{+}}(z)=D_{u}^{\pi^{-}}(z)=D_{\bar{u}}^{\pi^{+}}(z)=D_{\frac{\pi^{-}}{}}(z)=D_{s}^{\pi^{+}}(z)=D_{s}^{\pi^{-}}(z) \\
& =D_{\bar{s}^{+}}^{\pi^{+}}(z)=D_{\bar{s}}^{\pi^{-}}(z)=D_{c}^{\pi^{+}}(z)=D_{c}^{\pi^{-}}(z)=D_{\bar{c}}^{\pi^{+}}(z)=D_{\bar{c}}^{\pi^{-}}(z) .
\end{aligned}
$$

In case of the fragmentation into $D$-mesons we have

$$
D_{1}^{D}(z)=D_{c}^{D}(z)=D_{\bar{c}}^{D}(z)
$$

The fragmentation of any other flavour into a $D$ meson is strongly suppressed. The only relevant contributions come from $s+W^{+} \rightarrow D^{+}$, or $D^{0}$. Also we set

$$
\int_{0.2}^{1} D_{1}^{D}(z) d z \approx 1
$$

because each c-quark fragments in at least one charmed hadron. Furthermore the fragmentation functions are assumed to be spin independent. The form of the fragmentation functions is taken from [14]. For electromagnetic interactions the semi inclusive pion asymmetries are the most interesting quantities because of the large counting rates. For $\mathrm{CC}$ asymmetries this is not the case, because the tested asymmetries are basically the same as for purely inclusive reactions. (This is due to the fact that for up and down quarks weak isospin and strong isospin coincide.) For the single $W$ asymmetries we get

$$
\begin{aligned}
A^{W-, \pi^{-}} & :=\frac{d \sigma_{\uparrow \downarrow}^{\pi^{-}}-d \sigma_{\uparrow \uparrow}^{\pi^{-}}}{d \sigma_{\uparrow \downarrow}^{\pi^{-}}+d \sigma_{\uparrow \uparrow}^{\pi^{-}}} \\
& =\frac{\Delta u+\Delta c-(y-1)^{2}(\Delta \bar{d}+\Delta \bar{s})+\eta(z)\left(\Delta c-(y-1)^{2} \Delta \bar{s}\right)}{u+c+(y-1)^{2}(\bar{d}+\bar{s})+\eta(z)\left(c+(y-1)^{2} \bar{s}\right)}
\end{aligned}
$$




$$
\approx A^{W^{-}}\left(1+\eta(z)\left[\left(\frac{\Delta c}{G_{W^{-}}}-\frac{c}{F_{W^{-}}}\right)-(y-1)^{2}\left(\frac{\Delta \bar{s}}{G_{W^{-}}}+\frac{\bar{s}}{F_{W^{-}}}\right)\right]\right),
$$

with

$$
\begin{aligned}
t & =\tan ^{2} \theta_{C} \\
\eta(z) & =\left(D_{2}^{\pi}(z)-D_{1}^{\pi}(z)\right) \frac{1-t^{2}}{D_{1}^{\pi}(z)+t^{2} D_{2}^{\pi}(z)}, \\
F_{W^{-}} & =u+c+(y-1)^{2}(\bar{d}+\bar{s}) \\
G_{W^{-}} & =\Delta u+\Delta c-(y-1)^{2}(\Delta \bar{d}+\Delta \bar{s}) .
\end{aligned}
$$

Analogously, in the case of $W^{+}$exchange we obtain

$$
\begin{aligned}
A^{W^{+}, \pi^{+}} & :=\frac{d \sigma_{\uparrow \downarrow}^{W^{+}, \pi^{+}}-d \sigma_{\uparrow \uparrow}^{W^{+}, \pi^{+}}}{d \sigma_{\uparrow \downarrow}^{W^{+}, \pi^{+}}+d \sigma_{\uparrow \uparrow}^{W^{+}, \pi^{+}}} \\
& =\frac{-\Delta \bar{u}-\Delta \bar{c}+(y-1)^{2}(\Delta d+\Delta s)+\eta(z)\left(-\Delta \bar{c}+(y-1)^{2} \Delta s\right)}{\bar{u}+\bar{c}+(y-1)^{2}(d+s)+\eta(z)\left(\bar{c}+(y-1)^{2} s\right)} \\
& \approx A^{W^{+}}\left(1-\eta(z)\left[\left(\frac{\Delta \bar{c}}{G_{W^{+}}}+\frac{\bar{c}}{F_{W^{+}}}\right)+(y-1)^{2}\left(-\frac{\Delta s}{G_{W^{+}}}+\frac{s}{F_{W^{+}}}\right)\right]\right)
\end{aligned}
$$

with

$$
\begin{aligned}
F_{W^{+}} & =\bar{u}+\bar{c}+(y-1)^{2}(d+s), \\
G_{W^{+}} & =-\Delta \bar{u}-\Delta \bar{c}+(y-1)^{2}(\Delta d+\Delta s) .
\end{aligned}
$$

For our numerical simulations we integrated the fragmentation functions over $0.2<$ $z<1$, which proves to be a good compromise between high statistics and a good ratio of leading to non-leading particles. For the parametrization of the fragmentation functions we used

$$
D_{1}^{\pi}(z)=0.665 \frac{(1-z)^{1.75}}{z}, \quad D_{2}^{\pi}(z)=\frac{1-z}{1+z} D_{1}^{\pi}(z)
$$

which is an improved fit along the line of [15] to the newer data from [14]. Fig. 3 shows the differences $A^{W^{+}, \pi^{+}}-A^{W^{+}}$and $A^{W^{-}, \pi^{-}}-A^{W^{-}}$. In those figures we use the set LO "standard scenario" from [11] which does not contain any contribution 
from the charm quarks. As shown in fig. 1, the anticipated accuracy for HERA CC events is of the order of per cent while the signal in fig. 3 is of the order of per mille. Thus the differences $A^{W^{+}, \pi^{+}}-A^{W^{+}}$, and $A^{W^{-}, \pi^{-}}-A^{W^{-}}$are too small to be useful as a new signal for the nucleon spin decomposition. However, it offers an urgently needed opportunity to test one of the basic underlying assumptions, crucial for all semi inclusive signals, namely the spin independence of the fragmentation functions. Obviously this assumption could be tested at the percent level with CC pion asymmetries.

The situation for charm production is much more favourable. The resulting formulas for single asymmetries read

$$
\begin{gathered}
A_{W^{-}}^{D}:=\frac{d \sigma_{W^{-}, D}^{\uparrow \downarrow}-d \sigma_{W^{-}, D}^{\uparrow \uparrow}}{d \sigma_{W^{-}, D}^{\uparrow \downarrow}+d \sigma_{W^{-}, D}^{\uparrow \uparrow}}=-\frac{\Delta \bar{s}+t^{2} \Delta \bar{d}}{\bar{s}+t^{2} \bar{d}} \\
A_{W^{+}}^{D}:=\frac{d \sigma_{W^{+}, D}^{\uparrow \downarrow}-d \sigma_{W^{+}, D}^{\uparrow \uparrow}}{d \sigma_{W^{+}, D}^{\uparrow \downarrow}+d \sigma_{W^{+}, D}^{\uparrow \uparrow}}=\frac{\Delta s+t^{2} \Delta d}{s+t^{2} d}
\end{gathered}
$$

These asymmetries are obviously a direct signal for the strange and antistrange polarization and fig. 4 shows that the anticipated statistical accuracy for $\mathcal{L}=1000 \mathrm{pb}^{-1}$ should allow for a very precise experimental determination. Even for $\mathcal{L}=100 \mathrm{pb}^{-1}$ the signal still allows to estimate the size of $\Delta s$ and $\Delta \bar{s}$. For the high luminosity $\mathcal{L}=1000 \mathrm{pb}^{-1}$ it even appears feasible to detect possible differences between $\Delta s(x)$ and $\Delta \bar{s}(x)$ which could be induced by the fact that a proton emits more readily virtual $K^{+{ }^{\prime}} s$ than $K^{-1} s$. We demonstrated that it is possible to measure at a polarized HERA with good accuracy both inclusive and semi inclusive CC spin asymmetries. Already for $100 \mathrm{pb}^{-1}$ one should obtain a good determination of $\Delta u_{V}$ and $\Delta d_{V}$ and a rough estimate for $\Delta s$ and $\Delta \bar{s}$, the latter for semi inclusive $D$ production. For $\mathcal{L}=1000 \mathrm{pb}^{-1}$ semi inclusive $D$ production is an especially interesting observable . We conclude also, that semi inclusive pion asymmetries offer a very good opportunity to test the assumed absence of spin effects in string fragmentation. 


\section{Acknowledgement}

This work grew out of the HERA-Workshop program 1995/96 at DESY. The discussion of polarized CC events was actually started by J. Kalinowski, M. Anselmino, and P. Gambino [10,16]. For the inclusive single $W$ boson asymmetries which both

groups calculated the results agree. This work has been supported by DESY, GSI, and BMBF. A.S. also thanks the MPI für Kernphysik in Heidelberg for support. 


\section{REFERENCES}

[1] HERA Workshop, 'Future Physics at HERA' 1995/96, proceedings to be published end of 1996.

[2] M. Veltri, M. Düren, K. Rith, L. Mankiewicz, A. Schäfer, in: Physics at HERA, vol. $1^{*} 447$ (1991) p. 447.

[3] L. L. Frankfurt, M. I. Strikman, L. Mankiewicz, A. Schäfer, E. Rondio, A. Sandacz, and V. Papavassiliou, Phys. Lett. B 230 (1989) 141.

[4] J. A. Bartelski, Acta Phys. Pol. B 10 (1979) 923.

[5] D. A. Dicus, Phys. Rev. D 5 (1972) 1367.

[6] M. Anselmino, P. Gambino and J. Kalinowski, Z. Phys. C 64 (1994) 267.

[7] J. Blümlein, N. Kochelev, preprint hep-ph/9603397, DESY-96-040 (1996).

[8] M. Maul, B. Ehrnsperger, E. Stein, A. Schäfer, preprint hep-ph/9602377, UFTP404-1995 (1996).

[9] J. Feltesse, private communication.

[10] J. Kalinowski, talk given at HERA Workshop: Future Physics at HERA 1996.

[11] M. Glück, E. Reya, M. Stratmann, W. Vogelsang, Phys. Rev. D 53 (1996) 4775.

[12] T. Gehrmann, W. J. Stirling, Phys. Rev. D 53 (1996) 6100.

[13] S. Güllenstern, M. Veltri, P. Gornicki, L. Mankiewicz, and A. Schäfer, Phys. Lett. B 312 (1993) 166.

[14] The European Muon Collaboration, Nucl. Phys. B 321 (1989) 541.

[15] J. J. Aubert et al., Phys. Lett. B 160 (1985) 417. 
[16] M. Anselmino, P. Gambino and J. Kalinowski, preprint DFTT 44/96, IFT-96-16, MPI-PhT/96-63 (1996). 


\section{Figure Captions}

Figure 1: Asymmetries $A^{W^{+}}$and $A^{W^{-}}$in the $Q^{2}$ range $600-1000(\mathrm{GeV})^{2}$, and $1000-$ $\max (\mathrm{GeV})^{2}$ for the luminosity of $100 \mathrm{pb}^{-1}$ using the parton distributions of [11].

Figure 2: Asymmetries $A^{W^{+}}$and $A^{W^{-}}$in the $Q^{2}$ range 600-max $(\mathrm{GeV})^{2}$, comparison between valence and sea quark contributions using set "Gluon C, leading order " [12 and "standard scenario, leading order" [11].

Figure 3 Asymmetry differences $A_{W+}^{\pi+}-A_{W+}$ and $A_{W-}^{\pi-}-A_{W-}$ in the $Q^{2}$ range 600-max $(\mathrm{GeV})^{2}$ using "standard scenario, leading order" [11].

Figure 4 Charmed asymmetries $A_{W+}^{D}$ and $A_{W-}^{D}$ in the $Q^{2}$ range $600-m a x(\mathrm{GeV})^{2}$ using "standard scenario, leading order" [11]. The error bars are given for the luminosity of $1000 \mathrm{pb}^{-1}$. 


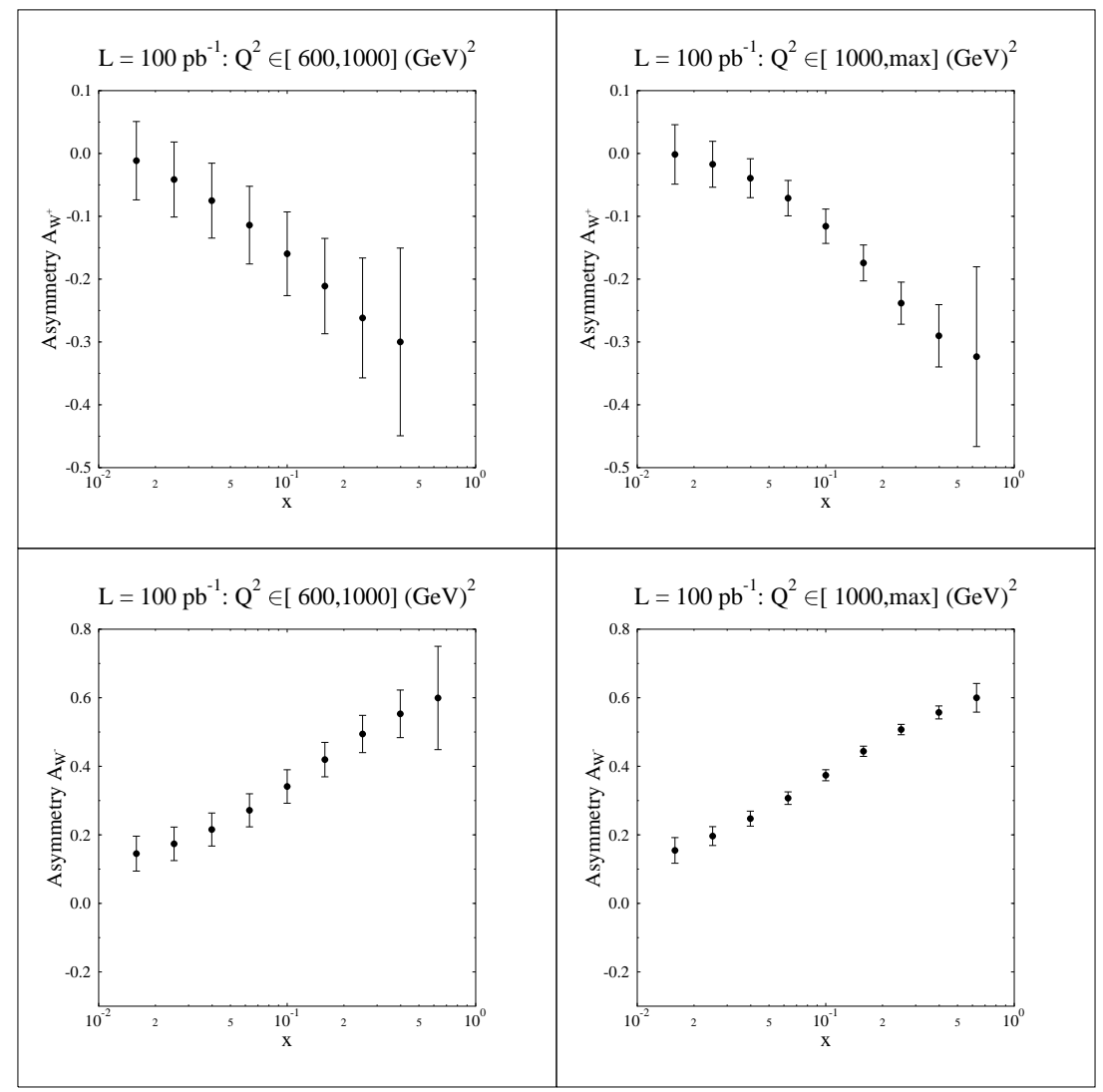

Figure 1 


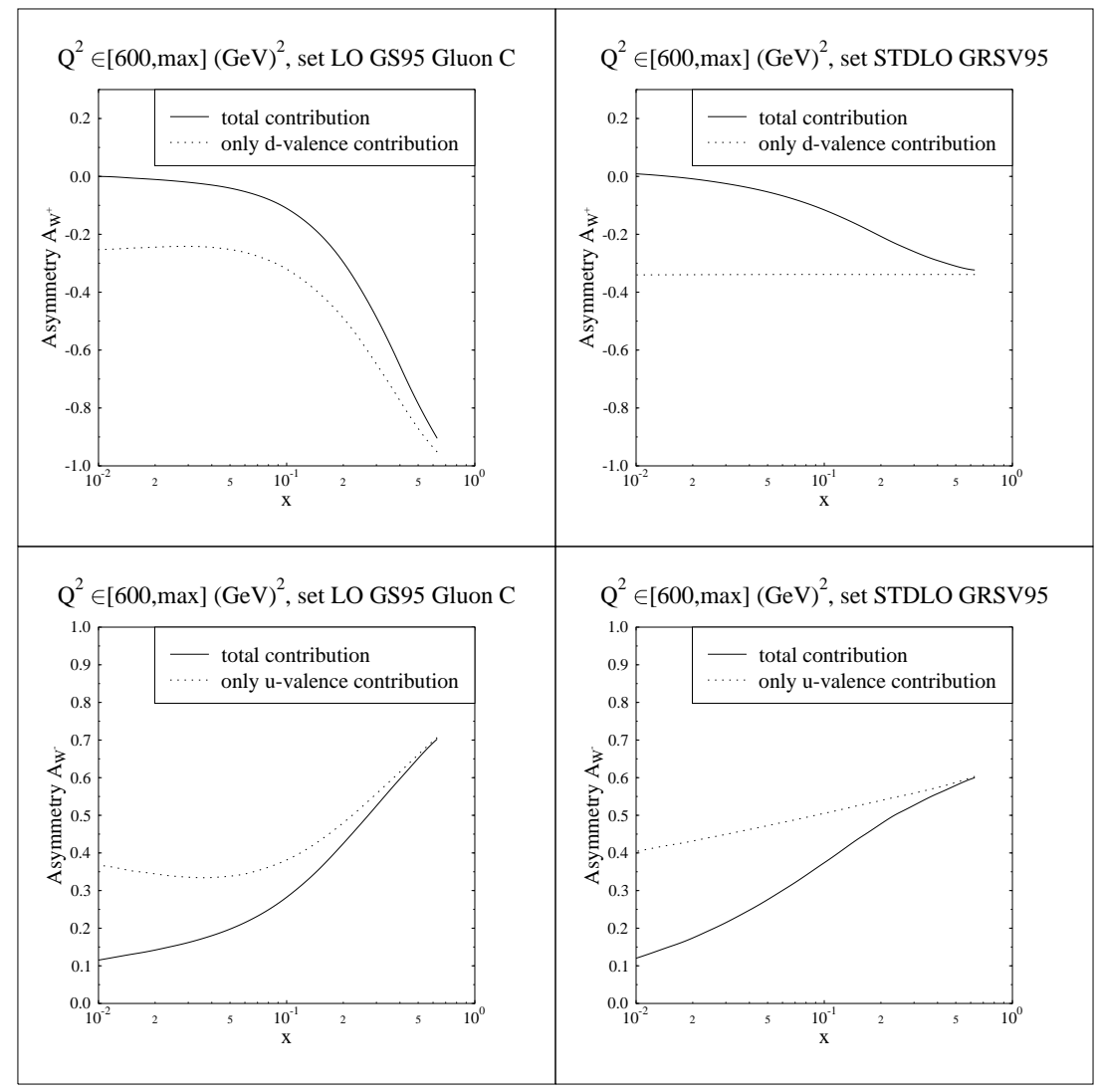

Figure 2 


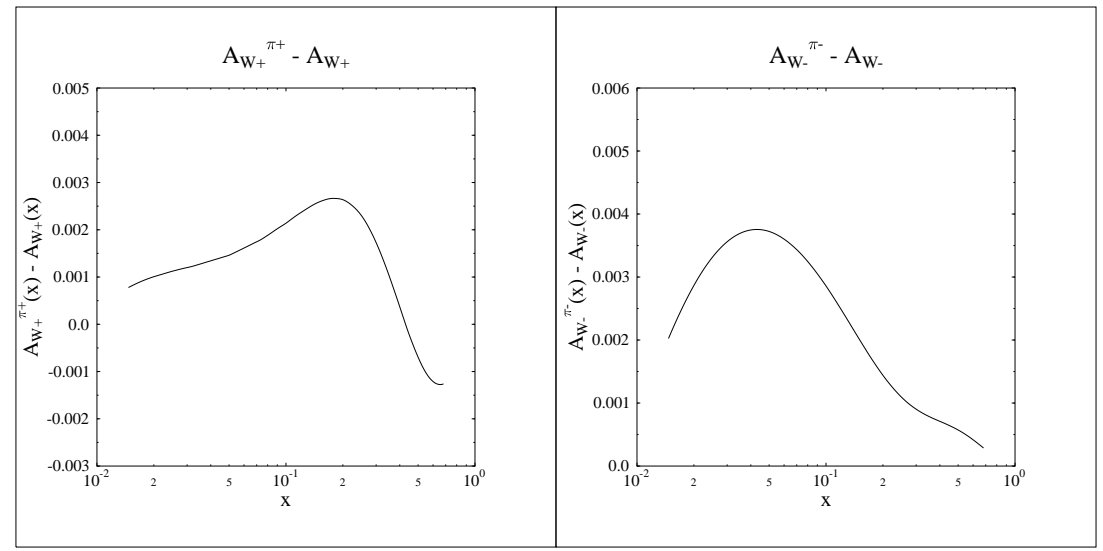

Figure 3

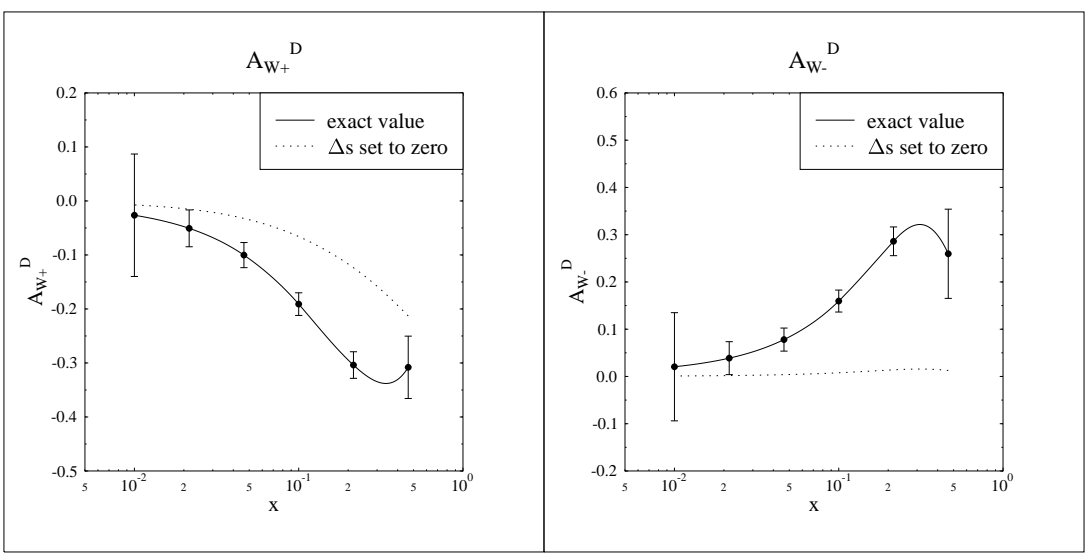

Figure 4 